keen to continue sponsoring activities that result in greater use of their services?

The tailored package of care and support for individual patients that has been created in some areas in response to the HIV epidemic would serve as a model for the care of patients with other chronic conditions. This system is threatened in three ways: general practitioners will be reluctant to take on patients with HIV infection or AIDS because they need costly drugs and create a heavy workload; acute and community services in the same district will be managerially separate and their relationships complicated by cross charging; and community care teams will have to agree contracts with districts of residence before crossing boundaries to facilitate continuity of expert care for patients.

In outline these are some of the problems for one specialty. Nevertheless, there may also be opportunities to modify the original plans to make them more workable. Indeed, the contracting process could be used to improve the service for patients with AIDS. If regional health authorities contract for AIDS services directly they could programme development to "roll out" new knowledge and skills from the few specialist centres to non-teaching districts. Centres with academic and research contracts could continue their research and development with extra regional funding to allow for their more complex case mix. "Satellite" districts would then hold contracts with the specialist centre for a form of shared care. These would be based on protocols, negotiated between clinicians, specifying the level of work to be handled in each district if the patient was willing. An additional strategy would be for consultants from the specialist centres to take on sessions with other district clinics, and vice versa. Just as important, the contracts could also specify the training commitments necessary for systematically disseminating knowledge and skills. Some such mechanism is vital as the numbers of patients will soon become too great for just a few centres to manage.

It is a great worry that the NHS review may threaten the quality and quantity of provision of services for genitourinary medicine and HIV infection. Urgent further thought must be given to supporting their open access systems for reasons of personal and public health. Not only must they survive, but they must be encouraged to develop and expand to meet the rapidly growing demands for their complex services. Otherwise many clinics and departments will contract that increasingly prevalent strain of AIDS - the acute income deficiency syndrome - which in the free market world has a negligible survival rate.

CHRIS BENTLEY Senior Registrar MICHAEL W ADLER Professor of Genitourinary Medicine

Academic Department of Genitourinary Medicine,

University College and

Middlesex School of Medicine,

London WIN 8AA

Department of Health. Working for patients. Contracts for health services: operating contracts. London: HMSO, 1990.

2 Department of Health. HIV and AIDS: resource allocations 1990/91. London: DoH, 1990 $(\mathrm{EL}(90) \mathrm{P} / 30$.

3 Working group to examine workloads in genitourinary medicine clinics. Report. London: DoH 1988. (Chairman A Monk.)

4 Department of Health. District allocations. London: DoH, 1990. (EL(90)MB/22.

5 Department of Health. Working for patients: WP2 funding and contracts for hospital services. London HMSO, 1989.

6 Department of Health. Contracts for health services: operational principles. London: DoH, 1989.

\title{
Children with diabetes
}

\section{Every health district should have a specialist clinic for their care}

The ravages of diabetic complications are not seen in a children's diabetes clinic. Nevertheless, they haunt the paediatrician who believes that sustained control of the blood glucose concentration will protect today's child from dying 20 years hence with renal failure, partially blind, and with ulcerated neuropathic feet. ${ }^{1}$

Diabetes in a child pervades all aspects of family life. The essential components of care are straightforward enough balancing insulin and diet with the day's activities - but the orchestration of the life of the diabetic child with the aim of achieving near normal glycaemia is a demanding business for all concerned: the child, the family, and the clinic team. Might the burden of care be lightened by more specialised attention and might normoglycaemia then be achieved, offering a better deal for adult life? A recent study by a working party of the British Paediatric Association goes some way towards answering these questions. ${ }^{2}$

Swift et al sent a questionnaire to all 820 consultant paediatricians who were members of the British Paediatric Association in March 1988 to study the services for children with diabetes in Britain. Replies were received from $93 \%$ of the consultants, giving information from 205 of the 220 health districts or boards. The answers showed that though the services for children with diabetes were well organised in some districts, there were substantial deficiencies in the network of care. Just over one quarter of districts did not have a designated specialist clinic for children with diabetes; $19 \%$ of consultants worked in districts with no support from diabetic nurse specialists or health visitors (and most only had such nursing support to a marginal degree); $9 \%$ of clinics did not provide regular measurements of glycated haemoglobin or fructosamine concentration; $63 \%$ did not provide the support of a trained paediatric dietitian; and $30 \%$ did not have a dietitian in attendance. In $13 \%$ of clinics children were seen regularly by senior house officers, and $45 \%$ of clinics did not offer out of hours support by telephone from specialist nurses or consultants for the children's families.

The scene is patchy and falls short of standards recently published by the British Diabetic Association for the specialist supervision of children with diabetes. ${ }^{3}$ The working party's report identifies the paediatricians who can be regarded as specialists in diabetes: they run large clinics specifically for diabetic children and their families. It is in association with these 100 specialist clinics that we find, predictably perhaps, the additional services of specialist nurses or health visitors, with a dietitian and a psychologist in attendance; a clinic stall with books and British Diabetic Association information packs; an active local parents group; arrangements for liaison with transfer of care to the counterpart adult clinic; and the routine practice of measuring blood pressure, screening for proteinuria, and examining the eyes.

Furthermore, clinical practice in the management of children with diabetes is changing in the wake of applied clinical research. New tests are beginning to define the early markers of diabetic complications in children, such as measures of heart rate variability, blood pressure response to 
posture, and pupil adaptation to the dark. ${ }^{4}$ Such additional measurements will in this next decade become components of good clinical practice in children's diabetes clinics and are likely to constitute the starting points and end points of therapeutic trials such as the evaluation of multiple injection regimens. ${ }^{56}$ This additional range of clinical measurements will demand well organised, well equipped, and well staffed specialist clinics. Meanwhile the family and child may judge that their hallmark of quality care is not achieving normoglycaemia nor the detection of early markers of diabetic disease - they may be more concerned with support, information, and empowerment as partners in care with open telephone access to members of their clinic team to talk through domestic diabetic emergencies.

However the quality of care is defined the British Paediatric Association working party argues the case that modern management of diabetes is complex and requires the services of a fully trained specialist team. The 1990 s will inevitably see an increase in the complexity of the care needed for children with diabetes as data on latent diabetic disease and new therapeutic interventions intrude on to the routine clinic scene. Children's diabetes clinics that are staffed by paediatricians trained in diabetes management will be essential to each district general hospital-in the same way as specialist neonatal intensive care units run by paediatricians trained in neonatal medicine are essential today. The British Paediatric Association report sets the scene: the immediate deficiencies in service should be addressed now; each district should provide a fully staffed specialist clinic for children with diabetes and their families. Otherwise, in the future the purchasers of health care and dissatisfied families will have good reason to take their custom elsewhere.

Professor of Child Health,

J D BAUM

Institute of Child Health,

Royal Hospital for Sick Children,

Bristol BS2 8BJ

1 Deckert T, Poulsen JE, Larsen M. Prognosis of diabetics with diabetes onset before the age of 31 Diabetologia 1978;14:363-70.

2 British Paediatric Association Working Party. The organization of services for children with diabetes in the United Kingdom: report of the British Paediatric Association Working Party. Diabetic Med 1990;7:457-64.

3 British Diabetic Association Committee. What professional supervision should children with diabetes and their families expect? London: British Diabetic Association, 1989.

4 Clarke CF, Piesowicz AT, Spathis GS. Pupillary size in children and adolescents with type diabetes. Diabetic Med 1989;6:780-3.

5 Smith CP, Dungar DB, Mitten S, et al. A comparison of morning and bedtime ultralente administration when using multiple injections in adolescence. Diabetic Med 1988;5:352-5.

6 McCaughey ES, Betts PR, Rowe DJ. Improved diabetic control in adolescents using the Penject syringe for multiple insulin injections. Diabetic Med 1986;3:234-6.

\section{Shoulder pain in the elderly}

\section{Common and mostly easily treated}

Two recent community studies have shown that at least a quarter of elderly people have shoulder pain. ${ }^{12}$ Many accept this pain and disability without complaint, ${ }^{3}$ yet in most cases the cause is soft tissue lesions that are treatable in the surgery.

What are the common shoulder disorders? Among 644 elderly patients taken at random from general practice age-sex registers examination identified a shoulder disorder in 138 patients, 98 of whom had soft tissue lesions: rotator cuff tendinitis (45 cases), rupture (27), impingement (22), and frozen shoulder (four). ${ }^{1}$ Osteoarthritis was common in the acromioclavicular joint but rare in the glenohumeral joint, and there were also cases of rheumatoid disease and Milwaukee shoulder. When no shoulder lesion was found cervical spondylosis was the commonest cause of shoulder pain. A recent paper in the $B M \mathcal{F}$ made similar observations and concluded that if general practitioners included assessment of the shoulder in their screening of elderly patients many would benefit. $^{2}$

Assessment requires some knowledge of the anatomy of the shoulder. The glenohumeral joint is stabilised and controlled by the encircling rotator cuff mechanism. The subscapularis muscle lies in front and the supraspinatus above, with the tendon of the long head of the biceps in between. The infraspinatus muscle lies above and behind the head of the humerus; the teres minor lies behind. The subacromial bursa separates the upper part of the cuff from the hard undersurface of the acromion.

Repeated entrapment of the rotator cuff under the acromion produces soft tissue damage, usually affecting the supraspinatus but occasionally the infraspinatus or the bicipital tendon. Injury or overuse may provoke an acute lesion with oedema, haemorrhage, and inflammation. Repeated episodes lead to chronic tendinitis and sometimes dense calcification of the tendon. Eventually the cuff tears, and radiographs may show upward displacement of the head of the humerus. Other patients develop acute calcific tendinitis with a sudden onset of pain and almost total loss of movement, which usually resolves spontaneously. By contrast, frozen shoulder is characterised by an insidious onset of constant pain; gradual loss of movement in all directions; and slow, often incomplete, recovery over one to three years. ${ }^{4}$ Plain radiographs and routine blood tests are unhelpful, but frozen shoulder is associated with diabetes mellitus, thyroid dysfunction, hemiplegia, myocardial infarction, and cardiac surgery.

The site and character of the pain and its relation to movement may suggest the diagnosis. Rotator cuff tendinitis classically causes pain in the mid-range of abduction (the painful arc), sometimes with pain at rest. Disease of the acromioclavicular joint causes local tenderness and pain at the extremes of movement and when lying on the shoulder. Pain radiating down the arm and unrelated to movement is usually referred from the neck. Local metastases and apical carcinoma of the lung may also cause shoulder pain.

The full range of movement, both active and passive, should be assessed on both sides to see whether scapular movement is compensating for restriction of the glenohumeral joint. Palpation may show local tenderness, particularly over the acromioclavicular joint or the greater tuberosity. Any pain on neck movement should be noted. Specific muscles can be tested: resisting abduction with the arm abducted to $90^{\circ}$ and flexed to $30^{\circ}$ with the thumb pointing downwards tests the supraspinatus; and resisting external rotation from neutral with the elbow against the trunk tests the infraspinatus. Complete rupture of the cuff usually prevents active abduction beyond the mid-range and leads to wasting of the supraspinatus or infraspinatus.

Acute lesions are treated by rest and analgesic or antiinflammatory drugs followed by remobilisation with simple exercises to improve the range and strength of movement. Local injection with a depot preparation of steroids may improve symptoms considerably, but is probably best 